\title{
Reverse Total Shoulder Arthroplasty
}

\section{Editorial Comment}

\author{
Guido Marra MD, Xavier Duralde MD
}

Published online: 16 July 2011

(C) The Association of Bone and Joint Surgeons (R 2011

The reverse total shoulder arthroplasty (RTSA) represents the most ground-breaking development in the world of shoulder surgery since the introduction of arthroscopy. Based on Paul Grammont's design changes and biomechanical principles $[1,2]$, modern RTSA has provided an option to both restore function and relieve pain in a large group of patients in whom there has been no other viable treatment option. Earlier attempts to create such a prosthesis in Europe and the United States were met with failure due to high loosening rates, component dissociation, and fracture. Widespread use of the RTSA was likely delayed in the United States owing to design deficiencies and failures of the early designs utilized in the United States during the 1960s. Despite a relatively brief clinical experience with this device in the United States, the European experience is now reaching 20 years. This experience has been critical in evolving the surgical technique and evaluating medium-term results.

In this symposium, we attempt to cover the reverse prosthesis from all angles. We have included a history of the failed attempts to create a reverse prosthesis that preceded Paul Grammont's revolutionary design [2]. Another historical article provides Grammont's guiding design principles and sheds light on the early evolution of this prosthesis [1].

Clinical papers in this symposium begin with the evaluation of patients with cuff tear arthropathy, as well as the

\section{G. Marra $(\bowtie)$}

Department of Orthopaedic Surgery, Loyola Medical Center,

2160 S 1st Avenue, Suite 1700, Maguire Center,

Maywood, IL 60153, USA

e-mail: gmarra@lumc.edu

\section{Duralde}

Peachtree Orthopaedic Clinic, Atlanta, GA, USA

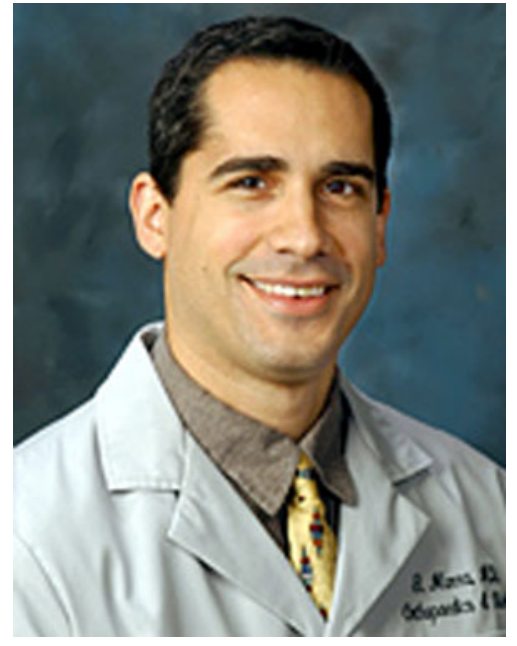

Fig. 1 Dr. Guido Marra is shown.

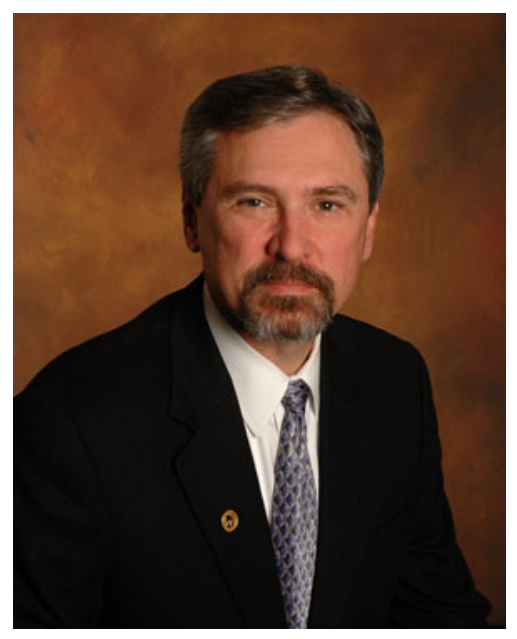

Fig. 2 Dr. Xavier Duralde is shown. 
radiographic progression of this degenerative process. A biomechanical analysis of the reverse prosthesis provides the reader with an understanding of the basic biomechanical principles of the prosthesis, as well as the challenges faced by designers and surgeons. We explore the results of the reverse prosthesis based on diagnosis, including traditional diagnoses of cuff tear arthropathy, arthritis with cuff tear, and rheumatoid arthritis. We also present early data on the results of RTSA applied to less traditional diagnoses of fractures, tumors, and revision arthroplasty. The most common complications related to RTSA, including scapular notching and instability, are reviewed by some of the world's most experienced surgeons.

As with any new technology, surgeons and researchers have begun moving into the next phase of understanding and employment of RTSA. This includes advances in the prosthetic design itself and refining surgical techniques based on observed treatment outcomes and complications. The best amount of lateralization of the joint line is now being re-evaluated to maximize external rotation strength while limiting notching and glenoid baseplate loosening.
This symposium represents a snapshot of the state of the RTSA in 2011. The papers here represent work by the world leaders in RSTA. We would like to thank all of the authors for their hard work in bringing their insights and latest data on this developing topic. This is a truly international effort, with reports from the world leaders in Europe, Asia, and America. The reader will note a substantial number of unanswered questions remain. Ongoing research by this group and others will continue to perfect Grammont's original theories and prosthetic design for the betterment of patients with complex shoulder problems.

\section{References}

1. Baulot E, Sirveaux F, Boileau P. Grammont's Idea: The Story of Paul Grammont's Functional Surgery Concept and the Development of the Reverse Principle. Clin Orthop Relat Res. 2011 January 6 [Epub ahead of print].

2. Flatow EL, Harrison AK. A History of Reverse Total Shoulder Arthroplasty. Clin Orthop Relat Res. 2011 January 7 [Epub ahead of print]. 